\title{
Large-scale analysis of transcriptional cis-regulatory modules reveals both common features and distinct subclasses

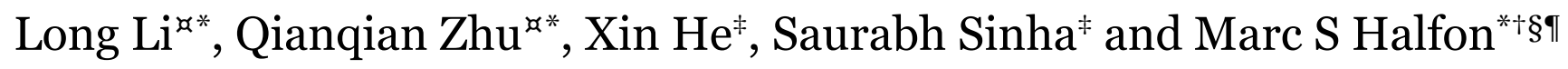

Addresses: *Department of Biochemistry, State University of New York at Buffalo, Buffalo, NY 14214, USA. ${ }^{\star}$ Department of Biological Sciences, State University of New York at Buffalo, Buffalo, NY 14214, USA. *Department of Computer Science, University of Illinois Urbana-Champaign, Urbana, IL 61801, USA. §New York State Center of Excellence in Bioinformatics and the Life Sciences, Buffalo, NY 14203, USA. "Department of Molecular and Cellular Biology, Roswell Park Cancer Institute, Buffalo, NY 14263, USA.

$\bowtie$ These authors contributed equally to this work.

Correspondence: Marc S Halfon. Email: mshalfon@buffalo.edu

Published: 5 June 2007

Genome Biology 2007, 8:RIOI (doi:I0.I I86/gb-2007-8-6-rI0I)

The electronic version of this article is the complete one and can be found online at http://genomebiology.com/2007/8/6/RIOI
Received: II April 2007

Revised: 23 May 2007

Accepted: 5 June 2007

(c) 2007 Li et al; licensee BioMed Central Ltd.

This is an open access article distributed under the terms of the Creative Commons Attribution License (http://creativecommons.org/licenses/by/2.0), which permits unrestricted use, distribution, and reproduction in any medium, provided the original work is properly cited.

\begin{abstract}
Background: Transcriptional cis-regulatory modules (for example, enhancers) play a critical role in regulating gene expression. While many individual regulatory elements have been characterized, they have never been analyzed as a class.

Results: We have performed the first such large-scale study of cis-regulatory modules in order to determine whether they have common properties that might aid in their identification and contribute to our understanding of the mechanisms by which they function. A total of 280 individual, experimentally verified cis-regulatory modules from Drosophila were analyzed for a range of sequence-level and functional properties. We report here that regulatory modules do indeed share common properties, among them an elevated GC content, an increased level of interspecific sequence conservation, and a tendency to be transcribed into RNA. However, we find that dense clustering of transcription factor binding sites, especially homotypic clustering, which is commonly believed to be a general characteristic of regulatory modules, is rather a feature that belongs chiefly to a specific subclass. This has important implications for current computational approaches, many of which are biased toward this subset. We explore two new strategies to assess binding site clustering and gauge their performances with respect to their ability to detect all 280 modules and various functionally coherent subsets.
\end{abstract}

Conclusion: Our findings demonstrate that cis-regulatory modules share common features that help to define them as a class and that may lead to new insights into mechanisms of gene regulation. However, these properties alone may not be sufficient to reliably distinguish regulatory from nonregulatory sequences. We also demonstrate that there are distinct subclasses of cis-regulatory modules that are more amenable to in silico detection than others and that these differences must be taken into account when attempting genome-wide regulatory element discovery. 


\section{Background}

Regulated spatial and temporal control of gene expression is a fundamental process for all metazoans, and much of this regulation occurs through the interaction of transcription factors (TFs) with specific cis-regulatory DNA sequences. The best-defined of these regulatory elements are promoters, which are easily identified based on their position surrounding the transcription start sites (TSSs) of their associated genes [1]. However, promoters comprise just a small fraction of important functional cis-regulatory sequences. A large amount of gene regulation is mediated by cis-regulatory elements that are distal to the promoter and organized in a modular fashion (reviewed by [2]). Each module regulates a particular temporal-spatial pattern of gene expression that is a subpart of the entire expression pattern of its associated gene; at the molecular level, each contains a series of binding sites for a specific complement of TFs. Often referred to as 'enhancers', these elements can lie hundreds of kilobases away from the promoter and can be located 5', 3', or within the intron of their own or a non-associated gene. Here, we use the more generic term 'cis-regulatory module' (CRM) to refer both to enhancers and to other classes of regulatory sequences.

The number of CRMs in the genome is believed to be very high; Davidson [2] suggests that there might be five-to-ten times as many individual CRMs in the genome as there are genes. It has become increasingly apparent that polymorphisms and mutations in CRMs play a major role as producers of normal phenotypic variation, as inducers of birth defects and chronic diseases, and as a powerful evolutionary driving force [2-4]. Despite their prevalence and importance, however, much less is known about CRMs in general than about promoters. This is largely due to the difficulties involved in identifying CRMs, which until recently has been possible only through a dedicated empirical approach of testing sequence fragments for regulatory activity in a reporter gene assay, either in transgenic animals or an appropriate cell culture system. In the past several years, a number of computational approaches for CRM identification have been attempted, with varying degrees of success (for example, [522]). Broadly speaking, most of these methods fall into either or both of two classes: those based on sequence alignment, or those dependent on transcription factor binding site (TFBS) clustering. In the first, putative CRMs are predicted based on conservation of non-coding sequences between two or more related species. In the latter, CRMs are defined as regions containing a particular number and/or combination of specific TFBSs. Considerations regarding these approaches and their variations have been reviewed elsewhere [23-28] and will not be discussed at length here. However, it is important to note that all of these methods have at their core an underlying assumption that CRMs contain common properties that will facilitate their discovery, that is, interspecific conservation or TFBS clustering.
From numerous examples, we know that both of these assumptions at times hold true. Many known CRMs are wellconserved in related species [22,29,30], and most of the extensively studied CRMs, in particular the enhancers of the Drosophila early patterning genes, consist of a dense cluster of TFBSs containing multiple occurrences of TFBSs for a small number of transcription factors [31-33]. This latter property is sometimes referred to as 'homotypic clustering' of TFBSs due to the repeated numbers of similar sites [34]. Nevertheless, there are also characterized CRMs that do not contain one or the other, or even both, of these properties. Late pair-rule expression of the Drosophila runt gene, for instance, is regulated by a diffuse CRM spread over $5 \mathrm{~kb}$ of sequence that is poorly conserved in distantly related Drosophila species [35,36]. Although this is typically viewed to be the exception rather than the rule, evidence to support this belief is thin and suffers from significant ascertainment bias: since many known CRMs were discovered based on one of these two properties, there is naturally an overrepresentation of conserved CRMs with clustered TFBSs. Thus, the actual extent to which these are common or unusual CRM characteristics remains undetermined.

We recently constructed a database of cis-regulatory elements in Drosophila melanogaster, the REDfly database, which contains records for over 650 experimentally verified positive-acting CRMs drawn from the published literature [37]. These CRMs are responsible for regulating the expression of a diverse set of genes in many different tissues and stages of development. Here, we present the results of our first large-scale analysis of the REDfly CRMs to define properties that are common to CRMs as a class, and those that are present only in specific CRM subsets. In the first section of the paper we describe the general sequence properties of Drosophila CRMs and show that CRMs are more GC-rich and evolutionarily conserved compared to other non-coding sequences, and are likely to be transcribed into RNA. Our data indicate that while CRMs have these distinct common properties as a class, they are difficult to distinguish from non-CRMs as individual sequences. In the second part of the paper we focus on TFBS clustering and show that homotypic TFBS clustering is prevalent only in certain CRM groups. We also undertake two new approaches to CRM discovery, neither of which are biased by any prior knowledge of binding sites, and show that these too favor the subclasses of CRMs with the greatest amount of TFBS clustering. Throughout, we consider the impact of the unknown fraction of CRMs present in unannotated non-coding sequence on all aspects of CRM discovery and analysis.

\section{Results Basic characteristics of the REDfly CRMs} Number and size

At the time we initiated this study, the REDfly database [37] contained 544 records of known Drosophila CRMs. We chose 
for analysis the subset of these that were non-overlapping and that were less than 2,100 base-pairs (bp) in length. This length cutoff captured $75 \%$ of the non-overlapping CRMs and was imposed based on our concern that CRMs of greater than $2 \mathrm{~kb}$ of sequence or so would contain large amounts of nonfunctional sequence (that is, that a more minimal CRM would exist within the larger sequence that had not yet been experimentally isolated). There were 280 CRMs associated with 148 genes, with an average length of $760 \mathrm{bp}$ (Figure S1-1A in Additional data file 1 ), that met these criteria and are referred to hereafter as the 'REDfly analysis CRMs'. A detailed listing of these CRMs can be found in Additional data file 2. Analysis of a subset of these CRMs, in which only those $\leq 1$, ooo bp in length were used, gave essentially identical results to those reported below (data not shown).

\section{Functional roles}

In order to determine the breadth of the functional spectrum covered by the genes associated with the REDfly analysis CRMs, we looked at the Gene Ontology (GO) terms for these genes and at the stages and tissues in which the REDfly analysis CRMs regulate gene expression. GO term designations to which $\geq \mathbf{1 0} \%$ of the CRM-associated genes map are shown in Table S1-1 in Additional data file 1. Although there is a bias toward CRMs associated with genes encoding transcription factors $(>50 \%)$ and for genes involved in development (>80\%), embryonic, larval, and adult stages of development are all represented (Figure S1-1B in Additional data file 1). A large variety of tissues are also represented (Figure S1-1C in Additional data file 1). Of these, embryonic blastoderm is the most heavily covered tissue (19\%), followed by neuronal tissue (13\%). An alternative breakdown of tissue representations is provided in Figure S1-2 in Additional data file 1.

\section{Genomic location}

Figure S1-1D in Additional data file 1 describes the location of the REDfly analysis CRMs with respect to the TSS of their associated genes: $61 \%$ of the CRMs are located 5 ' to the annotated TSS; $13 \%$ of the CRMs overlap the promoter or are completely contained within the first 500 bp 5 ' of the TSS while $38 \%$ begin more than 500 bp $5 ' .13 \%$ of the CRMs are downstream of the annotated 3 ' end of their genes, while $16 \%$ lie within introns. The vast majority of these are within the first (50\%) or second (27\%) introns, but CRMs are found within sixth and seventh introns as well (Figure S1-3 in Additional data file 1).

Genes with multiple transcripts present a particular problem for assigning the location of CRMs; when the transcripts are generated from alternative promoters, a CRM can be upstream of one TSS, but in an intron of another. As a result, 10\% of the REDfly analysis CRMs have a 'mixed' upstream and intronic location. It is generally unknown whether the CRMs influence the expression of all or only a subset of the transcripts with which they are associated.

\section{CRMs have an elevated GC content}

We measured the average GC content of the REDfly analysis CRMs and compared it to that of coding sequences, intergenic regions, and introns (Figure 1). It has previously been shown that the GC content in coding sequences is higher than that of non-coding sequences [38,39], and that Drosophila promoters tend to be AT-rich [40]. Surprisingly, we found that the REDfly analysis CRMs have a higher average GC content than other intergenic or intronic sequence, although a lower GC content than coding regions (mean 0.45 (standard deviation (SD) 0.06) versus 0.37 (0.07), rank sum test $P<1 \mathrm{e}-16$; 0.45 (0.06) versus 0.54 (0.05), rank sum test $P<1 \mathrm{e}-16$ ). This does not appear to be the result of a higher density of TF binding sites present in the CRMs, as an analysis of the footprinted binding sites contained in the FlyReg database [41] shows that they have an average GC content similar to that in nonCRM intergenic sequence (data not shown). No differences in the results were observed when various tissue- or stage-specific subsets were used in place of the entire 280 REDfly analysis CRMs (data not shown). A moderate negative correlation exists between CRM length and GC content (Figure 2; Spearman's $\rho=-0.27, P<9 \mathrm{e}-06)$. Size-matched random non-coding sequences are uncorrelated with GC content (Figure 2b; Spearman's $\rho=0.03, P=0.28$ ). Assuming that longer introns are likely to contain more CRMs than short introns [42], the higher GC content of CRMs versus non-regulatory non-coding sequence may help to account for the observations by Haddrill et al. [43], who saw both a positive correlation between intron length and GC content, and a negative correlation between GC content and sequence divergence between D. melanogaster and D. simulans introns (as CRMs are more highly conserved; see below).

\section{CRMs are more highly conserved than non-regulatory sequences}

Functional sequences are expected to be conserved among related species, a property that has been used successfully for the identification of CRMs in many organisms (reviewed by [44]). This approach has worked particularly well in vertebrates, for which a wide range of related species have been sequenced. However, while it is clear that conserved sequences frequently contain CRMs, it is less clear how often CRMs lie in non-conserved sequences, nor how many conserved sequence regions do not contain CRMs. To begin to address these questions, we constructed pairwise alignments between the REDfly CRM sequences in D. melanogaster and $D$. simulans, D. yakuba, D. erecta, D. ananassae, D. pseudoobscura, D. mojavensis, and D. virilis (more closely to more distantly related, respectively; [45]) using DIALIGN [46]. DIALIGN was chosen due to its strong performance in a previous assessment of alignment of simulated non-coding sequences [47]. We assessed both the conservation of the CRM sequences themselves and the conservation of sequences up to $1 \mathrm{~kb}$ to each side of the CRM and compared these alignments with alignments of size-matched, randomly selected non-coding sequences. We assessed conservation in 


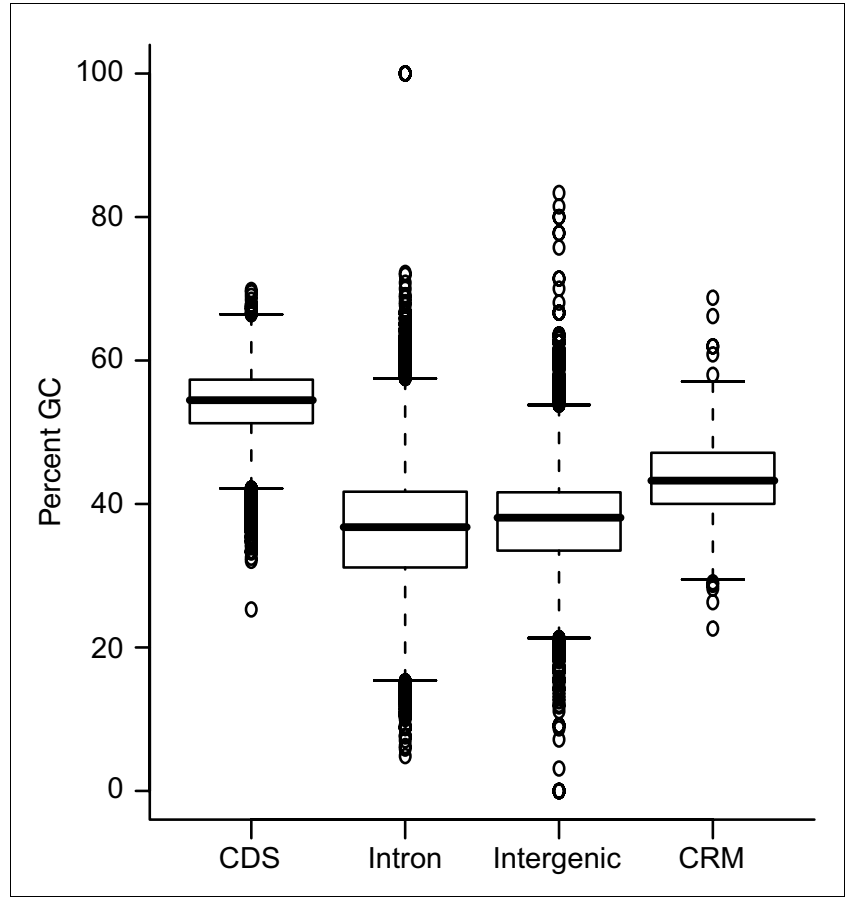

Figure I

GC content of the REDfly analysis CRMs as well as coding, intronic, and intergenic sequences.

terms of both fraction of aligned bases and degree of nucleotide identity between two sequences; both measures gave similar results (Figure 3; Figure S3-1 in Additional data file 3; data not shown).

We find that CRMs are on average significantly more wellconserved than randomly chosen non-coding sequences (Figure 3a; Figure S3-1 in Additional data file 3; KolmogorovSmirnov test, Bonferroni-corrected $P<7 \mathrm{e}-07)$. The sequences flanking the CRMs are generally less conserved than the CRMs but more conserved than the random sequences. Some of the increased conservation of the flanking sequences relative to randomly drawn ones may be due to the presence of coding regions within these sequences. However, this is unlikely to account for the entire observed difference as

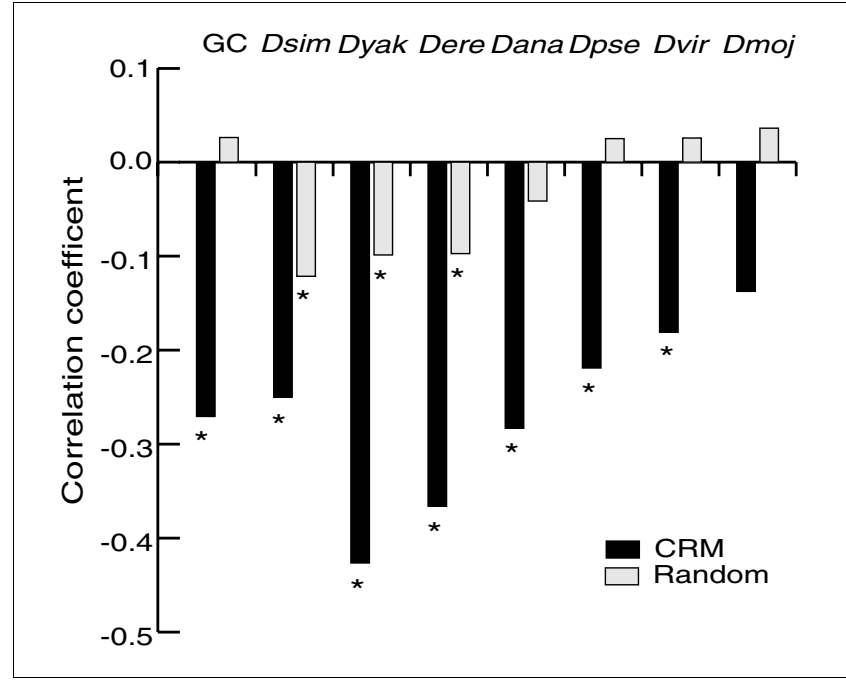

Figure 2

Correlations between CRM length and GC content (column I) and degree of sequence conservation with seven Drosophila species. Values given are the Spearman correlation coefficients. Black bars indicate CRM sequences, gray bars indicate size-matched randomly drawn non-coding sequence. Asterisks signify that the correlation is statistically significant (Bonferroniadjusted $P<0.05$ ). Dsim, D. simulans; Dyak, D. yakuba; Dere, $D$. erecta; Dana, D. ananassae; Dpse, D. pseudoobscura; Dvir, D. virilis; Dmoj, D. mojavensis.

the majority of the CRMs are sufficiently far from their associated coding regions that the flanking sequences contain only non-coding DNA (data not shown). We speculate that most of the difference is due either to a greater likelihood for the adjacent sequences to contain additional (as yet unidentified) CRMs, or to the gradual loss of regulatory function in these sequences due to binding site turnover (for example, [48-50]). Interestingly, we find that although as expected, the degree of CRM conservation decreases with increased evolutionary distance, the difference between the amount of conservation in CRMs versus random sequences remains essentially constant (Figure 3a). This is in marked contrast to the difference between coding and random sequences, which increases steadily with evolutionary distance. The different behaviors of the two types of functional sequences appear to

Figure 3 (see following page)

Sequence conservation properties of the REDfly analysis CRMs. (a) Average fraction of aligned bases between $D$. melanogaster and each of the other species for the CRMs (blue), CRM flanking sequences (green; \pm I kb to each side of the CRM; see text), coding regions (orange; based on 2,000 genes; see Materials and methods), and size-matched randomly selected non-coding sequences (red). Dashed lines indicate the $20 \%$ and $80 \%$ percentile values for the CRMs and random sequences. Also indicated are the 'differences' in conservation between CRMs and random non-coding sequences (black) and between coding sequences and random non-coding sequences (pink). Species abbreviations are as given in the legend to Figure 3. A similar graph showing the fraction of aligned 'identical' bases is given in Figure S3-I in Additional data file 3. (b) Histogram of the conservation fraction for CRMs (black bars) and random non-coding sequences (white bars) for $D$. melanogaster aligned with $D$. pseudoobscura. Histograms for the other species are shown in Figure S3-2 in Additional data file 3. (c) Median conserved block density for each of the species aligned to $D$. melanogaster. Blocks are defined as ungapped regions of seven or more nucleotides with $\geq 75 \%$ identity. Shown are block densities for CRMs (blue), CRM flanking regions (green), and size-matched randomly selected non-coding sequences (red). (d) Histogram of the distribution of conserved block density for CRMs (black bars) and random non-coding sequences (white bars) for D. melanogaster aligned with D. pseudoobscura. Histograms for the other species are shown in Figure S3-3 in Additional data file 3. 
(a)

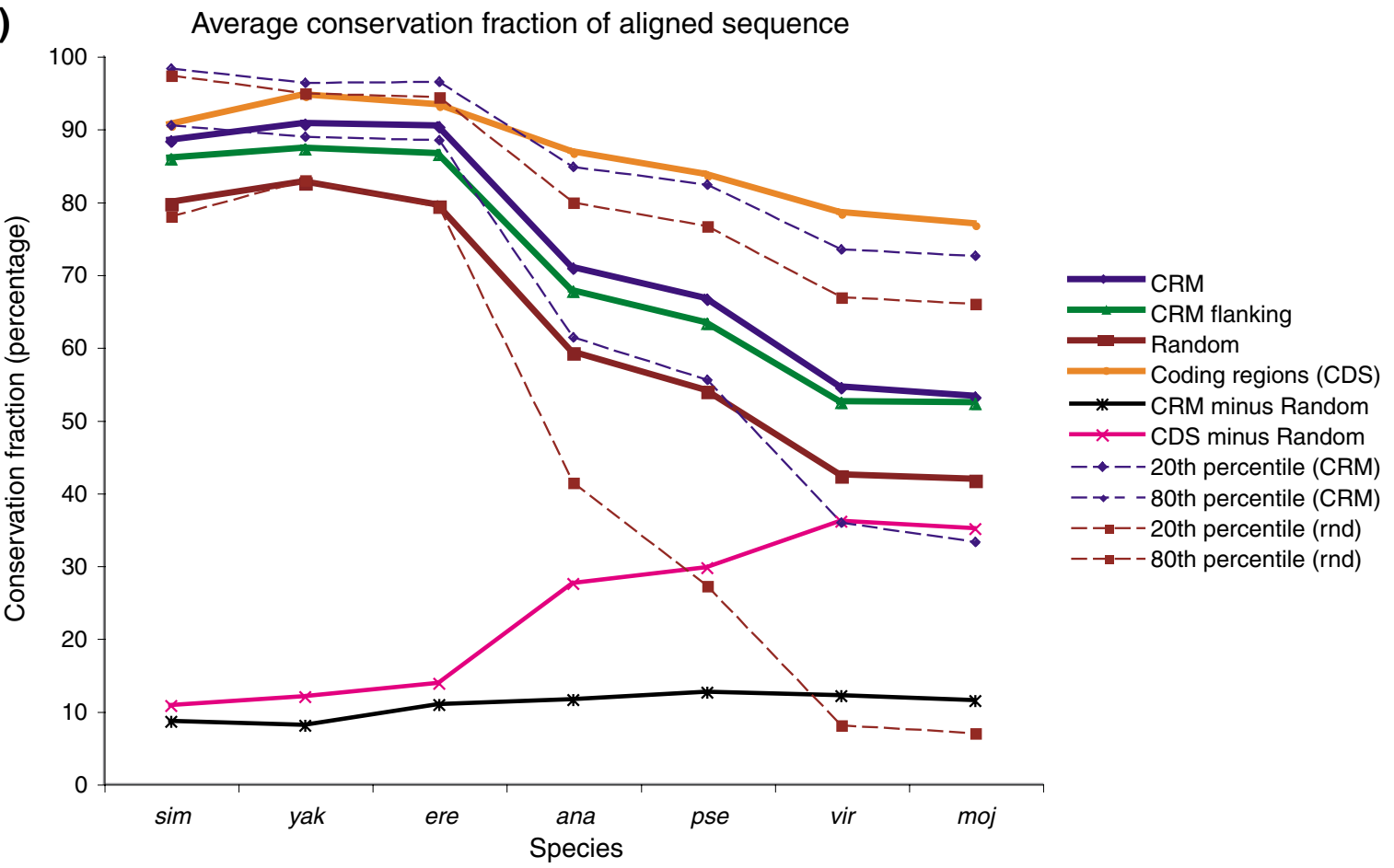

(b)

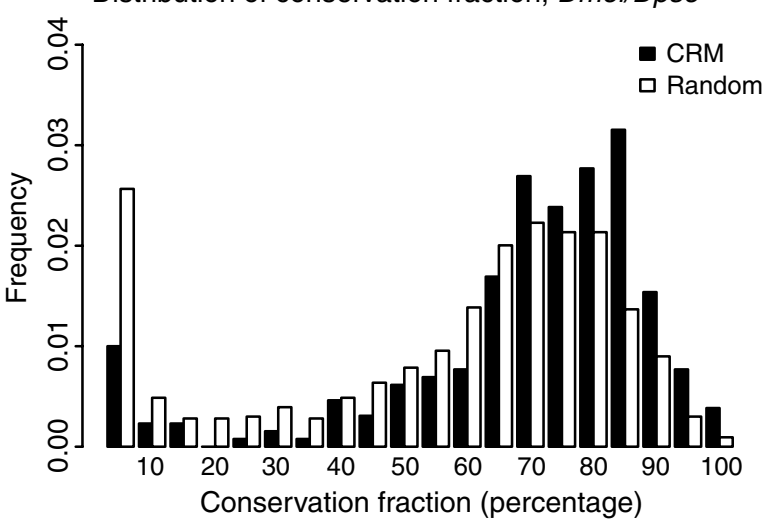

(d)

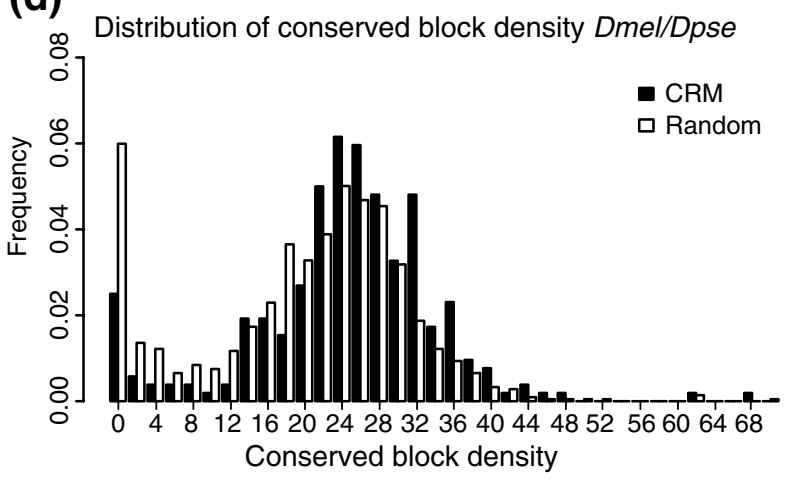

(c)

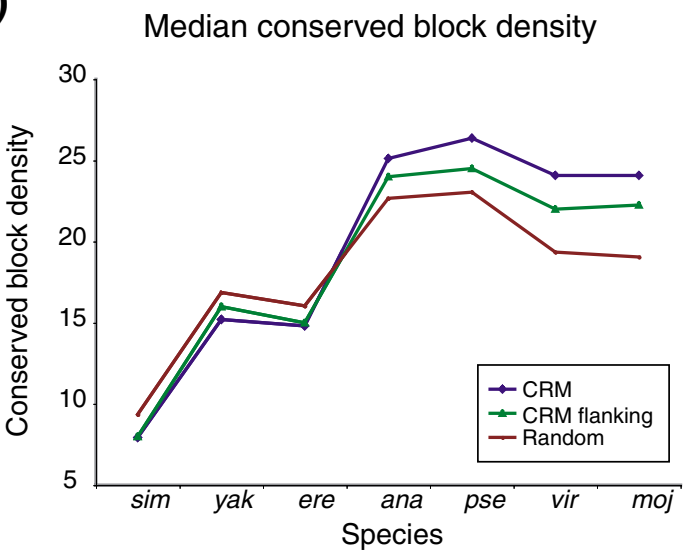

Figure 3 (see legend on previous page) 
be due to a faster rate of divergence in CRMs versus coding sequences. As with GC content, no differences in the results for any of the conservation-related properties were observed when various tissue- or stage-specific subsets were used in place of the entire set of 280 REDfly analysis CRMs (data not shown).

Despite the clear difference in mean conservation fraction between CRMs and random non-coding sequence, the distributions of the two sets are highly overlapping (Figure 3b; Figure $\mathrm{S}_{3}-2$ in Additional data file 3). Therefore, degree of sequence conservation would appear to be an ineffective way of reliably distinguishing regulatory from non-regulatory sequences. We note, however, that an unknown fraction of the random non-coding sequence we use will actually contain regulatory elements and might in addition contain other currently unannotated functional sequences such as missed first exons and micro-RNAs. The higher this fraction, the more likely we are to be underestimating the true amount of separation between the regulatory and non-regulatory sequences. We return to this point in more detail in the Discussion.

As we observed for GC content, CRM length and conservation fraction are negatively correlated, with more closely related species generally having a greater degree of correlation than more distantly related ones (Figure 2; $P<0.05$ ). We also observe a weak but statistically significant negative correlation for randomly selected non-coding sequences in the most closely related species. This is in contrast to results recently reported by Halligan and Keightley [51], who found that noncoding sequence length is negatively correlated with divergence. The difference may be due to the different scale of the two analyses: our study is mainly looking at much shorter sequences.

Although the magnitude of the difference in sequence conservation between CRMs and random non-coding sequences is relatively constant among all the analyzed species, the pattern of conservation differs. We looked at conserved sequence blocks of 7 bp or more with $\geq 75 \%$ identity in CRMs, their flanking sequences, and random non-coding sequences. While the length of conserved blocks does not vary significantly among these groups (with the exception of D. simulans; Figure S3-3 in Additional data file 3; data not shown), there is a significant difference in the density of conserved blocks in the more diverged species. In these species, CRMs have more blocks per kilobase than do random non-coding sequences (Figure 3c; Kolmogorov-Smirnov test, Bonferronicorrected $P<0.003$ ). As we saw for overall conservation, sequences adjacent to the CRMs fall in between the CRMs and the random sequences. Again, however, the distributions are highly overlapping, suggesting that conserved block density also is not a reliable discriminator between regulatory and non-regulatory sequences (Figure 3d; Figure $\mathrm{S}_{3}-4$ in Additional data file 3 ). Our results differ slightly from those of Papatsenko et al. [52], who observed an increased number of long (>2O bp) conserved blocks in CRM sequences when comparing $D$. melanogaster and D. pseudoobscura. The differences are likely due to the fact that that study defined blocks as having $100 \%$ identity versus our looser standard of $75 \%$ identity. Nevertheless, our overall conclusions are in agreement with those of Papatsenko et al. [52].

\section{Ultraconserved elements are overrepresented in CRMs}

Several recent studies have remarked on the presence of 'ultraconserved' elements and other highly conserved regions in both vertebrate and invertebrate genomes [19,53,54]. Ultraconserved elements (uc-elements) are long stretches of sequence $(\geq 50 \mathrm{bp}$ ) that are perfectly conserved over tens of millions of years of evolution. The majority of these are associated with genes encoding TFs and other regulators of development, and it has been hypothesized that uc-elements lying in non-coding regions might serve as all or parts of cis-regulatory modules [54]. Glazov et al. [55] have identified uc-elements conserved between $D$. melanogaster and $D$. pseudoobscura, and we examined the extent of overlap between these uc-elements and the REDfly analysis CRMs. Of the 20,301 non-coding uc-elements conserved between the two fly species, 84 overlap a REDfly analysis CRM by greater than $15 \mathrm{bp}$. On average, a mean of $98 \%$ (11\% SD) of each of these 84 uc-element sequences is contained within a CRM. In all, 61 of the REDfly analysis CRMs (22\%) contain at least one uc-element, with $28 \%$ of these containing two or more (Additional data file 4). This is significantly greater overlap than we find for uc-elements in size-matched random non-coding sequence controls ( $17 \%$ of sequence 'elements'; Fisher's exact $P<0.04)$. The overrepresentation of uc-elements within CRMs is even more apparent when the total amount of ultraconserved base-pairs is considered: $2.5 \%$ of the total REDfly analysis CRM sequence is ultraconserved, versus only $1.8 \%$ of size-matched random non-coding sequence (Fisher's exact $P$ $<2.2 \mathrm{e}-16$ ). Again, we note that these data are likely to understate the differences in the regulatory and non-regulatory populations due to the presence of an unknown number of regulatory and/or coding elements in the randomly selected sequence.

\section{CRM sequences are transcribed with high frequency} Recent transcriptional profiling studies using whole-genome tiled microarrays in a number of organisms have revealed that a much larger fraction of the genome than previously appreciated is transcribed into RNA [56-62] (reviewed by [63]). We used the microarray data of Manak et al. [64], which covers the Drosophila genome at 35 bp resolution, to determine whether or not the REDfly analysis CRMs are transcribed. We found that over 35\% (99/280) of the CRMs were transcribed versus only $23 \%(3,194 / 14,000)$ of size-matched randomly selected non-coding sequences $(P<4.05 \mathrm{e}-07$ by two-sample test of proportions). Thus, CRM sequences are transcribed with higher frequency than non-CRM sequences. Data from a second Drosophila tiled microarray experiment 
[58] are consistent with this result, although differences in microarray design prevent a direct comparison of the datasets (see Additional data file 5 , Table $\mathrm{S}_{5}-1$ and Figure $\mathrm{S}_{5}-1$ ).

\section{A modified Fluffy-tail test distinguishes CRM from non- CRM sequences}

We next turned our attention to a property often assumed to be common to the majority of CRMs, that of TFBS clustering. Abnizova et al. [65] have proposed a method, the Fluffy-tail test (FTT), that relies on homotypic TFBS clustering to identify CRMs. Like a number of other CRM discovery methods (for example, [34,66,67]), the FTT uses similar nucleotide subsequences as a proxy for related binding sites. The FTT score is based on the size of the largest group of 'similar words' - related nucleotide subsequences - in a CRM sequence and was reported to have excellent performance at distinguishing CRMs from non-regulatory non-coding sequences when analyzing 6o Drosophila CRMs (Figure S6-1 in Additional data file 6, columns 1 and 2). We therefore decided to make use of the FTT to test the underlying assumption that dense homotypic TFBS clustering is a general feature of CRMs.

We developed a revised version of the FTT, which we refer to as the FTT-Z (see Materials and methods), that performs similarly to the original test but eliminates a problem in which the score is confounded with the length of the sequence being analyzed (Figures S6-2 and S6-1 in Additional data file 6, columns 3 and 4 ). There are 41 of the REDfly analysis CRMs present in the original FTT training set. When we applied the FTT-Z to these 41 CRMs, we found that the separation between the CRMs and random non-coding sequence was very poor, suggesting that the FTT-Z score does not provide a good method for distinguishing regulatory from non-regulatory sequences (Figure 4, columns 1 and 2). However, there is a significant difference in the mean scores between the two groups (CRMs, $0.55 \pm 0.09$ (mean \pm standard error of the mean); random non-coding -0.01 \pm 0.07; rank sum test $P<$ 2.5e-05). We therefore went on to apply the test to all of the REDfly analysis CRMs. Once again, we found that the difference in the mean scores was statistically significant between CRMs and random non-coding sequences $(0.15 \pm 0.03$ versus $0.02 \pm 0.02$; rank sum test $P<0.02$ ), but the separation remained very poor (Figure 4 , columns 3 and 4 ).

\section{Blastoderm CRMs are different from other CRMs}

Although both sets of CRMs are significantly different from random sequence, the mean score when using all of the REDfly analysis CRMs is significantly smaller than the score using the 41 CRM training set (rank sum test $P<3.7 \mathrm{e}-04$ ). We noted that close to $80 \%$ of the $41 \mathrm{CRMs}$ are CRMs that regulate gene expression in the early embryonic blastoderm (referred to hereafter as 'blastoderm CRMs') and wondered whether this might account for the difference in scores. Therefore, we compared separately the 80 REDfly analysis CRMs annotated as being blastoderm CRMs and the remaining 200 non-blasto-

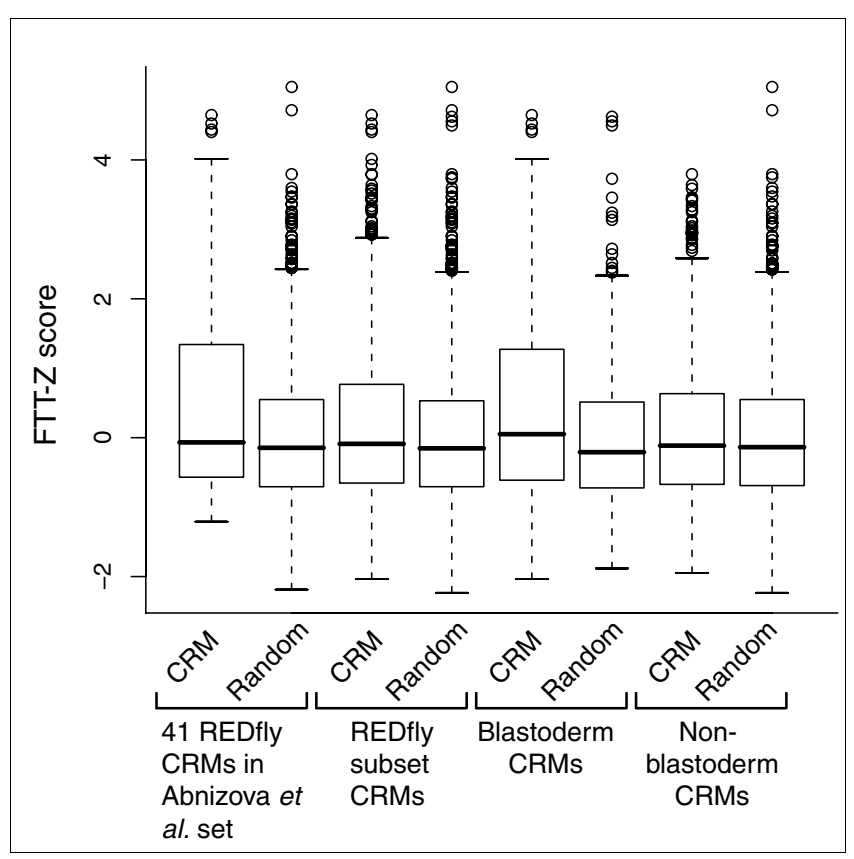

Figure 4

Results from the FTT-Z test. Boxplots indicate the median (heavy bar) and first and third quartiles of the data (boxed area). Details are provided in the text.

derm CRMs to both random non-coding sequence and to each other. While the blastoderm CRMs are significantly different from random sequence (Figure 4, columns 5 and 6 ; $0.36 \pm$ 0.06 versus $0.01 \pm 0.05$; rank sum test $P<8.2 \mathrm{e}-05$ ), the nonblastoderm CRMs and random sequence are indistinguishable (Figure 4, columns 7 and 8; $0.07 \pm 0.03$ versus $0.03 \pm$ o.o3; rank sum test $P<0.14$ ). Furthermore, the blastoderm and non-blastoderm CRMs are significantly different from one another (Figure 4, columns 5 and 7; rank sum test $P<$ 4.7e-04). We therefore conclude that the differences observed between the REDfly analysis CRMs and random non-coding sequences are due mainly to the presence of the blastoderm CRMs. These data suggest that although the blastoderm CRMs have large numbers of homotypic repeats, CRMs in general are no different from non-regulatory sequences in this regard.

We also tested whether stage- or tissue-specific categories of CRMs containing $\geq 15$ members (Figure $\mathrm{S} 1-\mathbf{1 B}$, C in Additional data file1) have FTT-Z scores that are different from randomly selected sequences. Other than the blastoderm CRMs, only those annotated as being associated with gene expression in the ectoderm, embryo, and adult have significant differences (Table S6-1 in Additional data file 6). However, these are not mutually exclusive classes, and the 'ectoderm' and 'embryo' CRMs overlap considerably with the blastoderm CRMs. Therefore, it is probable that the high FTT-Z scores of the blastoderm CRMs account for most of differences seen in these subsets. 
Table I

\begin{tabular}{lcc}
\hline $\begin{array}{l}\text { Performance of CRM discovery methods with respect to FTT-Z } \\
\text { score of confirmed CRMs }\end{array}$ & Reference & Median rank* \\
\hline Method & - & $50 \%$ \\
\hline Expected & - & $90 \%$ \\
Knownt & {$[6]$} & $92 \%$ \\
CIS-ANALYST & {$[67]$} & $73 \%$ \\
PFR-Searcher & {$[13]$} & $65 \%$ \\
Fly Enhancer & {$[33]$} & $57 \%$ \\
Ahab & {$[14]$} & $39 \%$ \\
- &
\end{tabular}

*Median rank of CRMs among all 280 REDfly analysis CRMs ranked by FTT-Z score. t'Known' CRMs are those used as training data by either/ or CIS-ANALYST or Ahab.

Biases in CRM type found by CRM discovery algorithms Sets of CRMs consisting primarily of blastoderm CRMs have been used to develop a number of computational approaches to CRM discovery [5,14,65-69]. Our results from the FTT-Z demonstrate that the blastoderm CRMs differ from CRMs in general in their degree of similar nucleotide subsequences. We therefore wondered if methods that were trained and tested on a blastoderm CRM dataset were biased toward discovery of CRMs with an unusually strong homotypic repeat structure. We reasoned that if this were the case, the CRMs found by these methods would have high FTT-Z scores, whereas unbiased methods would be uncorrelated with FTT$\mathrm{Z}$ scores. To test for such biases, we ranked all of the REDfly analysis CRMs by FTT-Z score and assessed the median rank (highest score $=100 \%$ ) of the CRMs discovered by the various other methods (Table 1). An unbiased method should have a median rank around 50\% ('expected' in Table 1), while a heavily biased method would have a median rank close to $100 \%$. We found that the previously known CRMs used in the training sets ('known') had a median rank of 90\%, confirming the heavy bias toward homotypic repeats in that set. Similarly, the CIS-ANALYST method of Berman et al. [6] predicted CRMs with a median rank of $92 \%$, suggesting that while effective for finding blastoderm-like CRMs with a dense subsequence repeat structure, this type of algorithm would be likely to perform poorly at discovering the majority of the known Drosophila CRMs. On the other hand, the Ahab algorithm used by Schroeder et al. [33] found CRMs with a median FTT$\mathrm{Z}$ rank of only $57 \%$ and might thus provide a CRM discovery method less geared toward the fraction of CRMs with highly repeated subsequences.

\section{A YMF-based method can distinguish CRMs from non- regulatory sequences}

As an alternative approach to addressing the question of whether binding site clustering is a general property of CRMs, we ran the motif-finding program YMF [70] for each CRM. YMF identifies motifs (words representing related subsequences) that are statistically overrepresented in a sequence or set of sequences and generates a count of how many unique motifs are found. The count of overrepresented motifs for each CRM was compared to the corresponding counts from 50 size-matched randomly selected non-coding sequences, and an empirically computed $P$ value was derived for each CRM (see Materials and methods). The resulting distribution of scores shows a significant bias towards low $P$ values, compared to the uniform distribution of $P$ values expected by chance (Figure 5a, blue versus red curves; Table 2; Kolmogorov-Smirnov test, $P<3.54 \mathrm{e}-11)$. This indicates that a CRM, on average, contains a larger number of significant motifs than a randomly chosen size-matched non-coding sequence. As a negative control, we created a collection of randomly chosen genomic sequences of the same lengths as the REDfly CRMs, and repeated the exercise. As expected, we found that the distribution of the $P$ value scores is close to uniform (Figure 5a, green curve; Table $2 ; P \cong 1$ ).

In light of the results from the FTT-Z indicating that the blastoderm CRMs have distinct properties, we recalculated the histogram of $P$ value scores (Figure 5 a) for each of several subsets of the REDfly analysis CRMs, formed on the basis of similarity of expression stages or tissue types (Table 2; Figure 5b). The blastoderm CRMs have a higher percentage of low $P$ values than the CRMs in general, consistent with the idea that TFBS clustering is more prevalent in this CRM subset $(P<$ 6.53e-04). Other tissue-specific subsets that were tested were not significantly different from random expectation (Table 2). One key difference from the FTT-Z results is that although the FTT-Z found that the non-blastoderm CRMs do not significantly differ from random non-coding sequences, these CRMs are still biased toward low YMF $P$ values and score in a range similar to the REDfly analysis CRMs as a whole (Figure $5 \mathrm{~b}$; data not shown). This difference is likely the result of the different ways each method assesses TFBS clustering (see Discussion).

Table 2

\begin{tabular}{lcc}
\multicolumn{3}{l}{ Significance of YMF results for tissue/stage-specific subsets } \\
\hline Tissue/stage* & Number of CRMs & $P$ value ${ }^{\dagger}$ \\
\hline All REDfly analysis CRMs & 280 & $3.54 \mathrm{E}-\mathrm{II}$ \\
Random non-coding & 280 & $\mathrm{I}$ \\
Blastoderm & $5 \mathrm{I}$ & $\mathbf{6 . 5 3 E - 0 4}$ \\
Non-blastoderm & 207 & $\mathrm{I.02E}-05$ \\
Mesoderm & 24 & 0.78 \\
Embryo & 128 & $9.00 \mathrm{E}-07$ \\
Non-embryo & 123 & 0.07 \\
Larva & 32 & $\mathrm{I}$ \\
Neuronal & 22 & $0.3 \mathrm{I}$ \\
\hline
\end{tabular}

*See Figure SI-I in Additional data file I). Only CRMs uniquely assigned to the tissue or stage are included here. HKolmogorov-Smirnov test. $P$ values for subsets are Bonferroni-corrected. Values in bold are significant. 


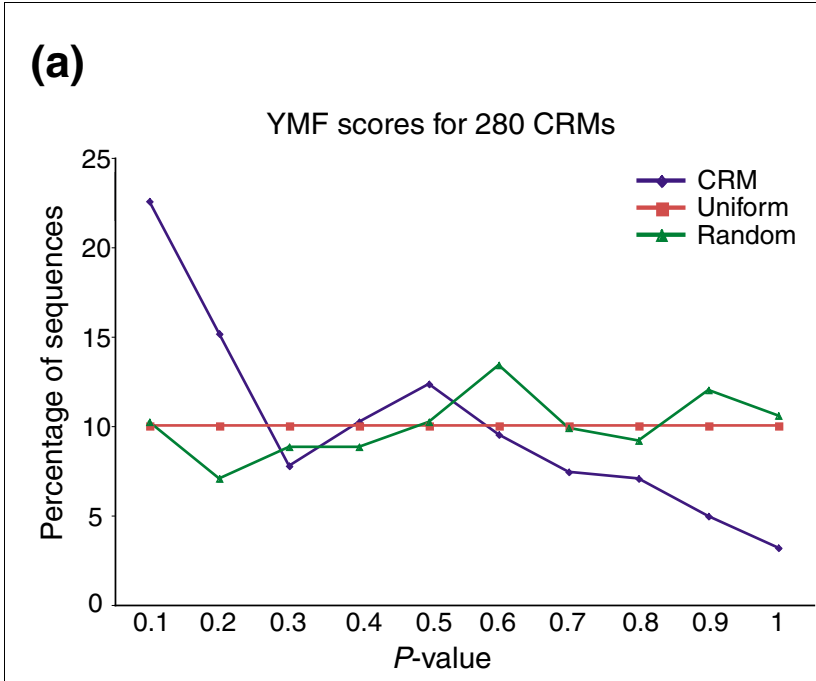

(b)

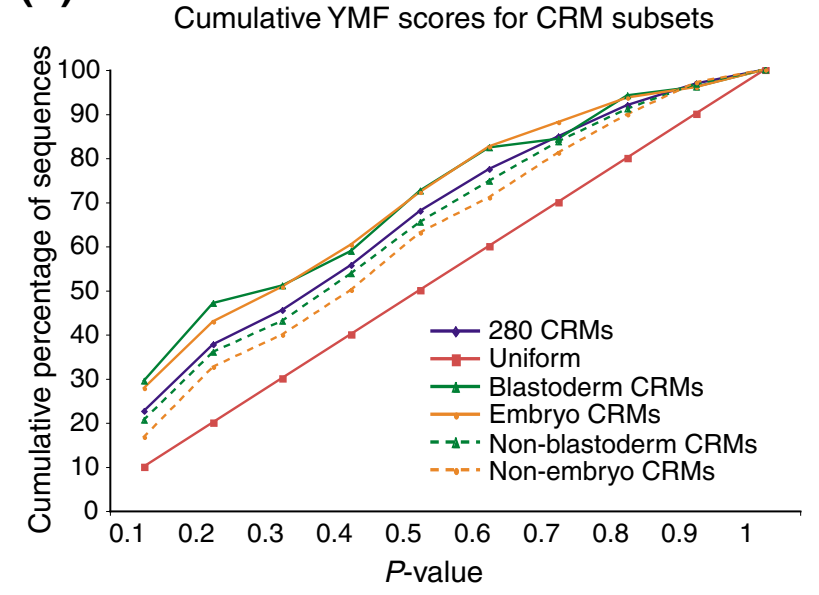

(c)

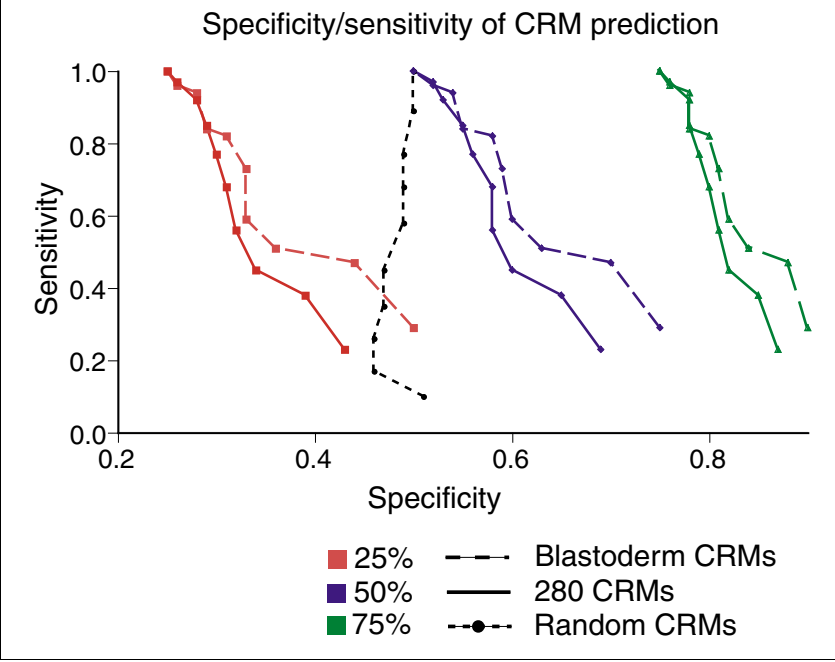

Figure 5

\section{Figure 5}

YMF scores for the REDfly analysis CRMs. (a) Histograms of percentage of CRMs for given $P$ value ranges (YMF scores). The histogram for all 280 REDfly analysis CRMs is shown in blue ('CRMs'), for randomly selected non-coding sequences in green ('Random'), and for the random expectation ('Uniform') in red. (b) Cumulative histograms of YMF scores for tissue- and stage-specific CRM subsets. The entire REDfly analysis set is shown in blue and the expected uniform distribution in red. Solid green lines indicate the blastoderm CRMs, while dashed green lines represent the non-blastoderm CRMs; orange solid and dashed lines show the embryo and non-embryo CRM subsets, respectively. Note that all subsets show significant deviation from the expected uniform distribution. (c) Specificity/sensitivity curves for CRM prediction using YMF. Three sets of curves are shown, representing three different assumptions as to the number of CRMs present in the randomly selected background sequences: 25\% CRMs (red), 50\% CRMs (blue), and 75\% CRMs (green). Solid lines indicate curves for the entire 280 REDfly analysis CRMs, while dashed lines show the blastoderm CRM subset. The black dashed line represents the curve for randomly selected sequences, shown for $50 \%$ background CRMs only. For each category, the random expectation is equal to the assumed number of CRMs in the background.

\section{Prediction of CRMs using YMF}

We can use the YMF $P$ value score to predict whether or not a given sequence is a CRM (see Materials and methods). Sensitivity of the prediction is based on the $P$ value score used as a threshold for calling a sequence a CRM, while the specificity of prediction depends on the true proportion of CRMs in the genome. That is, we assume that some number of the random non-coding sequences are in fact currently unidentified CRMs. Under the assumption that $50 \%$ of the input sequences are CRMs, we can achieve a prediction specificity of $69 \%$ at a sensitivity of $23 \%$, much better than the $50 \%$ specificity expected by chance. Figure $5 \mathrm{c}$ shows the specificity of CRM prediction expected at varying levels of sensitivity under different assumptions about genomic CRM abundance (25\%, $50 \%$, and $75 \%$ of randomly chosen genomic sequences being CRMs). Note that the blastoderm CRMs can be predicted with much better sensitivity/specificity than the other CRMs, consistent with our previous finding that they comprise a distinct CRM subclass (Figure 5c, dashed versus solid lines).

\section{Supervised learning and classification of CRMs versus random genomic sequences}

As a third way of testing the TFBS clustering properties of CRMs, we undertook a supervised learning approach to CRM classification based on a modification of the HexDiff algorithm [66]. We used frequencies of short subsequence words to train an algorithm to discriminate CRMs from non-CRMs (see Materials and methods). The classification accuracy was evaluated in a ten-fold cross validation exercise in which the REDfly analysis CRMs were treated as the positive set and an equal number of randomly chosen genomic sequences (of the same lengths as the CRMs) used as the negative set.

A set of 175 modules (the REDfly analysis set after removing CRMs $<500$ bp or $>2,000 \mathrm{bp}$ ), augmented with an equal sized 'negative' set of random sequences, could be classified correctly with an accuracy of $63.8 \%$ in a 10 -fold cross-validation 
Table 3

\begin{tabular}{lcc}
\hline \multicolumn{2}{l}{ Results from supervised learning } & \\
\hline Tissue/stage* & Classification accuracy & $P$ value \\
\hline REDfly analysis CRMs & $63.8 \%$ & $1.9 \mathrm{E}-0 \mathbf{7}$ \\
Blastoderm & $68.4 \%$ & $\mathbf{3 . 5 E}-03$ \\
Neuronal & $65.0 \%$ & 0.16 \\
Embryo & $59.1 \%$ & $\mathbf{0 . 0 4}$ \\
Larva & $42.5 \%$ & $\mathrm{I}$ \\
\hline
\end{tabular}

*See Figure $\mathrm{SI}-\mathrm{I}$ in Additional data file I. Only CRMs uniquely assigned to the tissue or stage are included here. $P$ values for subsets are Bonferroni-corrected. Values in bold are significant.

exercise (Table 3; Binomial test $P<1.9 \mathrm{e}-07$ ). Note that this figure is not comparable to the sensitivity or specificity values given for the YMF algorithm, since an accurate prediction in this exercise requires correctly classifying both 'positive' (CRM) and 'negative' (non-CRM) samples.

Like with the FTT and YMF methods, we also evaluated tissue- and stage-specific subsets of CRMs using this learning algorithm and a leave-one-out-cross-validation strategy. The 'blastoderm', and 'embryo' CRMs gave significantly high classification accuracy in similar cross-validation experiments (Table 3). As we saw with the other methods, the blastoderm CRMs have the most pronounced differences compared to the other CRM subsets and to the entire REDfly analysis set.

\section{Discussion}

Two commonly held assumptions about transcriptional cisregulatory modules are that their sequences are evolutionarily conserved and they contain a high degree of TFBS clustering. We present here a large-scale analysis of Drosophila CRMs designed to evaluate these and other CRM properties. This is the largest such study performed to-date for any metazoan; nevertheless, only about $1 \%$ of Drosophila genes are represented, with presumably only a subset of the CRMs for each gene. Our main conclusions can be summarized as follows: first, CRMs have distinct properties that as a group distinguish them from other types of DNA sequences, regardless of the tissues or stages in which they regulate gene expression. Second, these differences are typically not great enough to reliably classify a given unknown sequence as CRM or non-CRM. Third, TFBS clustering, and homotypic TFBS clustering in particular, can begin to provide more reliable classification of sequences as CRM or not CRM. Fourth, homotypic clustering is not a general characteristic of CRMs but rather is prevalent only in certain CRM subclasses.

\section{Sequence conservation}

Many CRMs, particularly in vertebrates, have been discovered by virtue of sequence conservation, leaving open the possibility that the strong conservation of CRMs noted in these species may be at least partially due to ascertainment bias. As the majority of the REDfly analysis CRMs were discovered by means other than an assessment of conservation (data not shown), they present a useful test set for evaluating this bias. Our results agree with studies of much smaller sets of Drosophila CRMs $[6,71]$. Similar to those, we see a statistically significant increase in the fraction of conserved sequence in CRMs versus non-CRMs, but with a distribution not too different from that of randomly selected sequences. One caveat lies in the fact that the REDfly CRMs are heavily biased toward those associated with genes with important functions in development, as there is evidence from studies in vertebrates that these CRMs are more likely to be conserved than others [29]. Overall levels of conservation of CRM sequences might thus be lower than what we have observed here.

The difference in degree of conservation between coding and non-coding sequences increases with evolutionary distance. Surprisingly, this is not the case for CRMs and their flanking sequences, both of which retain a roughly constant degree of difference in conservation fraction compared to random noncoding sequences. Thus, CRM sequences diverge more rapidly than coding sequences, but in proportion with the overall degree of sequence divergence of non-coding DNA. This may be due to a general conflation of CRMs and what we call random non-coding sequence: our CRMs might contain large amounts of non-regulatory non-coding sequence, or the randomly selected non-coding sequences might contain a large fraction of CRM sequence. We favor the view that both of these phenomena are occurring.

Support for the idea that the REDfly CRMs contain a substantial amount of non-regulatory sequence is provided by the negative correlations that we observe between CRM length and both GC content and sequence conservation. That is, longer CRMs are more like random non-coding sequences in their sequence properties than are shorter CRMs. We interpret this to mean that many of the REDfly CRMs are 'too long' - they have not been defined down to minimal functional sequences. However, we cannot rule out the (non-exclusive) possibilities that all of the CRM DNA is functional but either contains redundant elements that are more free to mutate, or constrained at a non-sequence level (for example, spacing between TFBSs).

\section{What fraction of non-coding sequence consists of CRMs?}

There is also good evidence to suggest that a significant fraction of the Drosophila non-coding DNA is functional and may harbor large numbers of CRMs. Halligan and Keightley [51] have recently estimated that greater than $50 \%$ of non-coding sequence is subject to selective constraint and, therefore, presumably functional, while Nelson et al. [72] have shown that genes with complex expression patterns are associated with longer flanking non-coding sequences than genes with simple expression patterns. Moreover, the Drosophila genome has a high rate of DNA loss in unconstrained sequences through 
deletion events [73]. Taken together, these data argue that the Drosophila genome is compact and contains a high proportion of regulatory sequence.

Both non-functional sequence included in our CRM set and, more importantly, a high density of CRMs within non-coding sequences, have important implications for the results we have presented here, as either feature will lead to underestimation of the observed sequence properties. That is, the more that our CRMs are contaminated with non-CRM sequence, and vice versa, the less good will be the separation that we detect between the two sequence classes. Therefore, although our results suggest that CRMs and non-regulatory non-coding sequences are not clearly distinguishable, an improved knowledge of the background fraction of CRMs in non-coding sequence would potentially reveal a greater separation. Unfortunately, until a truly unbiased empirical assessment of regulatory activity over an extensive selection of non-coding DNA is conducted, there may not be sufficient data to make a proper estimate of the true CRM fraction.

\section{Transcription of CRM sequences}

Whole-genome tiling microarray experiments and detailed EST sequencing projects have repeatedly revealed that much higher percentages of the genomes of multiple organisms are transcribed than originally believed [56-62], although the functional significance of this transcription remains unclear [63] and even controversial [74]. Our analysis suggests that a substantial number of intergenic and intronic transcribed sequences could be CRMs. Transcription of regulatory sequences has been observed previously, notably in the Drosophila bithorax complex (BX-C) and in the locus control regions (LCRs) of several vertebrate gene clusters [75-80]. Johnson et al. [81] suggest that transcription at the $\beta$-globin LCR is a consequence of RNA polymerase II recruitment by the LCR, but other studies suggest that active transcription of the CRM is required for gene activation (for example, [82]). In the BX-C, CRM transcription is restricted to the tissues in which the respective CRMs are active [75], but it is unknown whether or not most CRM transcription is temporally and spatially regulated or if it correlates with activity or inactivity of the CRM. Further study of transcribed CRM sequences at much higher spatial and temporal resolution than has currently been conducted will be necessary before these and related questions can be answered.

\section{TFBS clustering}

A commonly held assumption about CRMs is that they contain tightly clustered binding sites for one or several TFs, with most of the sites represented multiple times (that is, homotypically clustered) $[2,31]$. As there also exist examples of other types of CRM organization, an important question becomes determining which is the exception, and which the rule [83]. Since the REDfly CRMs span a broad range of regulatory systems, with most of the relevant transcription factors not characterized, we faced the technical challenge of assessing TFBS clustering without knowing the actual binding sites. We therefore used several different methods to count occurrences of similar words (motifs) as a surrogate for measuring the extent of TFBS clustering. The FTT looks only at the subsequence with the highest incidence in the CRM and thus provides a measure of homotypic TFBS clustering [65]. YMF, on the other hand, considers how many different motifs are overrepresented in the CRM [70]. YMF is, therefore, simultaneously a measure of homotypic clustering (each TFBS identified must be present at greater than background levels) and heterotypic clustering (multiple significant TFBSs must be present). Both methods clearly separate the blastoderm CRMs from the non-blastoderm CRMs, suggesting that not only do blastoderm CRMs tend to have more homotypic TFBS repeats than other CRMs (FTT results) but also that they frequently contain a larger number of distinct binding sites (YMF results). However, while non-blastoderm CRMs are indistinguishable from random non-coding sequences by the FTT, YMF clearly differentiates between the two. Heterotypic TFBS clustering may thus be a more common property of CRMs than extensive homotypic clustering, which appears to be a property mainly of specific CRM subclasses.

\section{Biological significance of homotypic clustering}

The prevalence of homotypic TFBS clustering in the CRMs responsible for regulating transcription in the early embryonic blastoderm may relate directly to the biology of early fly development. The use of CRMs consisting of multiple binding sites with varying affinities has long been recognized as an important component of the mechanism by which genes can determine their position with respect to a morphogen gradient $[84,85]$. This is precisely the situation found in the early fly embryo, which develops as a syncytium in which patterns of gene expression are largely determined by TF concentration gradients (reviewed by [86]).

Consistent with the idea that homotypic clustering is associated with interpreting positional information based on morphogen gradients, we note that a number of non-blastoderm REDfly CRMs associated with morphogen-responsive genes have high FTT-Z scores indicative of homotypic TFBS clustering. For instance, CRMs for the Ance/race gene, which responds to morphogen gradient signaling during embryogenesis [87], and for the salm and bi/omb genes, which respond to morphogen gradient signaling in the wing imaginal disc [88], rank in the top half of the FTT-Z scores (percent ranks $=75.2 \%, 69.1 \%$, and $54.1 \%$, respectively). Nevertheless, not all genes in these classes have high levels of TFBS clustering, suggesting that other means are also used to ensure correct readout of morphogen concentrations [89].

In circumstances where gene expression is not regulated through morphogen gradients, the need for dense TFBS clusters may be less important, consistent with our finding that homotypic clustering is not a general CRM property. In a cellular environment, TFs are unable to diffuse from one spatial 
gene expression domain to another as they do in the syncytial blastoderm, and sharp boundaries of TF activation domains can be maintained by other methods. The apparently more widespread presence of heterotypic TFBS clusters fits with the idea that transcriptional regulation is highly combinatorial, with CRMs acting to integrate the input from multiple signaling pathways and tissue-specific selector proteins [3,90].

\section{Implications for CRM discovery methods}

Our large-scale study of CRM sequences provides a starting point for re-evaluating existing methods for computational CRM discovery and designing new approaches. Most methods that have been developed for computational discovery of CRMs are based on the assumption that CRMs have common properties that can provide a signal for their identification, primarily either sequence conservation or TFBS clustering (see reviews in [23-28]). We have demonstrated here that CRMs do indeed share common properties, but the separation between the CRM and non-CRM populations is too poor to allow for successful discrimination. While this separation might be improved in organisms with a less highly compact genome than Drosophila, tests of conserved sequences in vertebrates also suggest that there, too, conservation alone is not a sufficient marker of regulatory sequence (for example, [91]). Nevertheless, despite not being enough to discriminate between CRMs and non-regulatory sequences on their own, these features can contribute to an overall scoring function for CRM identification.

Importantly, our results demonstrate that distinct subsets of CRMs can have specific properties not shared by all regulatory modules but which can be highly effective for CRM discovery. Therefore, one-size-fits-all approaches to CRM discovery are likely to be less effective than methods tailored toward specific subsets. A favorable strategy might therefore be to use methods that make use of a set of coexpressed genes for training data, such as the PFR-Sampler/Searcher programs [67], consistent with our finding that a supervised learning approach provided greater sensitivity than the other methods. Focusing on only a subset of CRMs might also make it easier to incorporate the detection or use of 'grammatical' rules, such as constraints in the spacing of TFBS pairs, into CRM discovery algorithms [9,11,92,93]. Our study also highlights the value of having a large and diverse set of known CRMs to use for training and evaluation purposes, something that for the higher eukaryotes currently exists only for Drosophila. Generating similar collections for human and other model organisms should remain an important future goal.

\section{Materials and methods Gene Ontology term mapping}

GO terms for the CRM-associated genes were determined using GOTermMapper [94].

\section{Sequences}

For comparisons of sequence conservation between CRMs and random non-coding sequences, five non-coding sequences of the same length as the CRM were randomly chosen from the genome of D. melanogaster for each CRM; values are the average value of the five sequences. Because Halligan and Keightley [51] have reported a correlation between the length of intergenic and intron sequences and their degree of conservation, it is possible that artifacts could be introduced if a CRM and its size-matched non-coding sequences were drawn from regions of different lengths. We tested this possibility for a small set of CRMs ( $n=30$, lengths $<1 \mathrm{~kb}$ different were considered identical) and found that there was no difference in the results if the random sequences were drawn from a similarly sized non-coding region or not $(P$ values for the various species ranged from 0.24-0.94 by paired $t$-test). Therefore, random sequences were chosen without regard to intergenic or intron sequence length. Conservation values for coding sequences are based on a random selection of 2,000 non-overlapping coding regions. Assignment of sequence as coding or non-coding was based on release 4.2 of the Drosophila genome annotation.

\section{Alignments}

Globally aligned sequence regions for each of the species used were obtained from the multiple alignments available from the Berkeley Comparative Genomics project [95] using the version 2 alignment of builds DroMel_4, DroYak_1, DroAna_20041206, DroEre_20041028, DroMoj_20041206, DroPse_1, DroSim_20040829, and DroVir_20041029. Sequence data for $D$. simulans and $D$. yakuba were produced by the Genome Sequencing Center at Washington University School of Medicine in St Louis [96], those for D. ananassae, D. erecta, D. mojavensis and D. virilis by Agencourt Bioscience [97], and those for D. pseudoobscura by the Human Genome Sequencing Center at Baylor College of Medicine [98]. 'Flanking' sequences were defined by extending each CRM (or size-matched random sequence) $1 \mathrm{~kb}$ on each end, or until the sequence could no longer be aligned, whichever was shorter. Each of these sequence regions was then aligned pairwise with the $D$. melanogaster sequence using DIALIGN [46] with the following parameters: -n -it -fa. Four of the REDfly analysis CRMs could not be cleanly assigned to orthologous sequence regions and were omitted from the assessments of conserved sequence (Dfd_EAE-C, gt_-1_construct, siz_loner/CG32434-PE, tld_promoterfusion).

\section{Transcribed CRMs}

Significance of CRM transcription was assessed using 50 sizematched sets of randomly selected non-coding sequence and a two-sample $z$-test of proportions such that: 


$$
z=\frac{p_{1}-p_{2}}{\sqrt{\left(\frac{1}{n_{1}}+\frac{1}{n_{2}}\right)(p)(1-p)}}
$$

where:

$$
p=\frac{p_{1} n_{1}+p_{2} n_{2}}{n_{1}+n_{2}}
$$

where $p_{1}=$ proportion of transcribed CRMs, $p_{2}=$ proportion of transcribed random sequences, and $n_{1}$ and $n_{2}$ are the total number of sequences for CRMs and random, respectively.

\section{FTT}

To reduce running time, the FTT MATLAB program [65] was rewritten in $\mathrm{C}++$. We created two versions of the FTT, one in which the GC content for the randomized ('shuffled') sequences was adjusted probabilistically (that is, each base chosen according to the probability of it being a specific nucleotide based on the nucleotide distribution of the original sequence), identical to the FTT of Abnizova et al. [65], and one in which GC content was held fixed by randomly rearranging the actual bases of the original sequences. Both of these versions performed identically and both gave similar results to the MATLAB version (data not shown).

The FTT-Z test was modified from the FTT as follows: for each CRM, the F score from the FTT was calculated as in the original program, except that we increased the number of shuffled sequences (' $r$ ') from $r=50$ to $r=1000$. To calculate the FTT-Z score, we then selected 500 randomly drawn noncoding sequences of the same length and from the same chromosome as the CRM (except in the case of CRMs on chromosome 4, for which random sequence was drawn from chromosome 2) and calculated the F score for each of these. A Z-score was then calculated as:

$$
\frac{F_{C}-\bar{F}_{R}}{\sigma_{R}}
$$

where $F_{C}$ is the F score of the CRM sequence and $\bar{F}_{R}$ and $\sigma_{R}$ are the mean and standard deviation of the F scores of the random sequences, respectively. Source code for the FTT-Z is available upon request.

For both the FTT and the FTT-Z, we conducted the tests a total of six times using either the CRMs or six independently generated sets of size-matched random non-coding sequences. The data reported in the text and in Figures 1 and 2 represent all six repetitions of the test.

\section{Computation of YMF score}

YMF [70] was run on every window of length 1,000 (with shifts of 100) in the given sequence, with motif length 6 and up to 1 degenerate symbol allowed. The count of motifs with a reported z-score $\geq 3$ is the 'YMF count score'. Random noncoding sequences of the same length were chosen from the D. melanogaster genome (Release 3.1), the YMF count score was computed for each of these, and an empirical $P$ value computed as the fraction of random sequences that scored greater than or equal to the given sequence's score. This empirical $P$ value is called the 'YMF score'. All sequences, including the randomly chosen ones, were tandem repeat masked with the 'Tandem Repeats Finder' program [99] with parameters '2 35801025 500' before processing.

\section{Estimation of sensitivity and specificity of CRM prediction}

A sequence is classified as a CRM if its YMF score (empirical $P$ value) is below a threshold $\tau$. Given a set of true CRMs, the sensitivity is simply the fraction of these classified as CRMs. The specificity of prediction is calculated by making assumptions about the fraction of randomly selected non-coding sequences that are actually CRMs, as follows. Let $\mathrm{S}$ be an input sequence, let $\mathrm{T}_{\mathrm{S}}$ be the event that $\mathrm{S}$ is classified as being a CRM (because its YMF score was below threshold), let $\mathrm{G}_{\mathrm{S}}$ be the event that $S$ is a true $C R M$, and let $B_{S}$ be the event that it is not a true CRM. The specificity is defined as the probability that a sequence $\mathrm{S}$ classified as a CRM is indeed a true CRM. That is, the specificity is given by:

$$
\operatorname{Pr}\left(G_{S} \mid T_{S}\right)=\frac{\operatorname{Pr}\left(T_{S} \mid G_{S}\right) \operatorname{Pr}\left(G_{S}\right)}{\operatorname{Pr}\left(T_{S} \mid G_{S}\right) \operatorname{Pr}\left(G_{S}\right)+\operatorname{Pr}\left(T_{S} \mid B_{S}\right) \operatorname{Pr}\left(B_{S}\right)}
$$

Note that $\operatorname{Pr}\left(T_{S} \mid B_{S}\right)=\tau$, since the probability that a random (non-CRM) sequence has an empirical $P$ value (YMF score) below threshold $\tau$ is itself $\tau$. $\operatorname{Pr}\left(T_{S} \mid G_{S}\right)$ is computed from the true (known) CRMs as the fraction of them that were classified as CRMs. Finally, we can make varying assumptions about $\operatorname{Pr}\left(G_{S}\right)$, the prior probability that a sequence $S$ is a $\mathrm{CRM}$, and set $\operatorname{Pr}\left(B_{S}\right)=1-\operatorname{Pr}\left(G_{S}\right)$ to obtain the specificity at threshold $\tau$. We show results under three different assumptions about the true proportion of CRMs: $\operatorname{Pr}\left(G_{S}\right)=0.25,0.5$, 0.75 .

\section{Classification of CRM versus random genomic sequences using supervised learning}

We implemented a variation of the HexDiff algorithm [66] to classify CRM sequences. The training data for our classifier consists of a set of CRMs ('positive' sequences) and a set of equally many random genomic fragments ('negative' sequences) with lengths matching the CRM lengths. (Sequences were not repeat masked for this exercise, and sequences with lengths $<500$ bp or $>2,000$ bp were excluded from this analysis.) We first find a set of discriminative words (hexamers) from the training data; the discriminative power $s(w)$ of a word $w$, is measured by:

$$
\ln \frac{f_{p}(w)}{f_{b}(w)}
$$


where $f_{p}(w)$ is the frequency of $w$ in the CRM sequences and $f_{b}(w)$ is the frequency of $w$ in the background, taken to be the entire release 3 Drosophila genomic sequence. After ranking all the words by this score, we choose a set $W$ to be the top $\kappa$ words, where $\kappa$ is determined from the training data (as described below). To score a sequence, we scan this sequence with a sliding window of size 500 and set the score of this sequence to be the maximum score of all windows. The score of a window is defined as:

$$
\sum n(w) s(w)
$$

for all $w$ in the set $W$, where $n(w)$ is the number of occurrences of $w$ in this window and $s(w)$ is the discriminative score we computed in the training stage. A sequence with score above some threshold $\tau$ is predicted to be a CRM. The values of $\kappa$ and $\tau$ are determined as those which, if used, lead to maximum prediction accuracy (least number of misclassified sequences) on the training sequences. In our experiments, we tried all values of $\kappa$ from 200 to 400 , with a step size of 50. Finally, each sequence in the test data is predicted as a CRM or not, using the values of $\kappa$ and $\tau$ learned from training data.

\section{Additional data files}

The following additional data are available with the online version of this paper. Additional data file 1 contains data on sequence-level properties of the REDfly analysis CRMs, including Figures S1-1 (basic properties of the CRMs), S1-2 (alternative mapping to tissues), S1-3 (distribution of intronic CRMs), and Table S1-1 (GO terms). Additional data file 2 is a GFFv3 file giving the locations and additional information on the REDfly analysis CRMs. Additional data file 3 contains Figures $\mathrm{S}_{3}-1$ through $\mathrm{S}_{3}-4$ with additional data on the evolutionary conservation of the REDfly CRMs. Additional data file 4 is a table showing the overlap of REDfly CRMs with ultraconserved sequences. Additional file 5 provides data on transcription of CRM sequences based on the microarray data of Stolc et al. [58] and includes Table S5-1 and Figure S5-1. Additional data file 6 illustrates differences between the original FTT and the revised FTT-Z (Figures S6-1, S6-2) and contains Table S6-1, which lists CRM subsets with significant FTT-Z scores.

\section{Acknowledgements}

We thank Casey Bergman and Mathieu Blanchette for comments on the manuscript, Jeffery Miecznikowski for advice on statistics, and Irina Abnizova for the FTT source code. LL performed the GC content, transcription, and FTT analyses. QZ performed the conservation studies and $\mathrm{XH}$ the supervised clustering. SS performed the YMF analysis, oversaw the supervised clustering, and provided general input into other aspects of the analysis. MSH coordinated the study, performed portions of the analysis, and wrote the manuscript. This work was supported by grants K22-HG002489 and P20-GM67650 from the NIH.

\section{References}

I. Smale ST, Kadonaga JT: The RNA polymerase II core promoter. Ann Rev Biochem 2003, 72:449-479.

2. Davidson EH: The Regulatory Genome: Gene Regulatory Networks in Development and Evolution Ist edition. Burlington, MA: Academic Press; 2006.

3. Carroll SB, Grenier JK, Weatherbee SD: From DNA to Diversity: Molecular Genetics and the Evolution of Animal Design 2nd edition. Malden, MA: Blackwell Publishing; 2005.

4. Wray GA: The evolutionary significance of cis-regulatory mutations. Nat Reviews Genet 2007, 8:206-2I6.

5. Berman BP, Nibu Y, Pfeiffer BD, Tomancak P, Celniker SE, Levine M, Rubin GM, Eisen MB: Exploiting transcription factor binding site clustering to identify cis-regulatory modules involved in pattern formation in the Drosophila genome. Proc Natl Acad Sci USA 2002, 99:757-762.

6. Berman BP, Pfeiffer BD, Laverty TR, Salzberg SL, Rubin GM, Eisen MB, Celniker SE: Computational identification of developmental enhancers: conservation and function of transcription factor binding-site clusters in Drosophila melanogaster and Drosophila pseudoobscura. Genome Biol 2004, 5:R6I.

7. Boffelli D, McAuliffe J, Ovcharenko D, Lewis KD, Ovcharenko I, Pachter L, Rubin EM: Phylogenetic shadowing of primate sequences to find functional regions of the human genome. Science 2003, 299: I39|-1394.

8. Castro B, Barolo S, Bailey AM, Posakony JW: Lateral inhibition in proneural clusters: cis-regulatory logic and default repression by Suppressor of Hairless. Development 2005, I 32:3333-3344.

9. Erives $A$, Levine $M$ : Coordinate enhancers share common organizational features in the Drosophila genome. Proc Natl Acad Sci USA 2004, I 0 I:385 I-3856.

10. Halfon MS, Grad Y, Church GM, Michelson AM: Computationbased discovery of related transcriptional regulatory modules and motifs using an experimentally validated combinatorial model. Genome Res 2002, 12:1019-1028.

II. Hallikas O, Palin K, Sinjushina N, Rautiainen R, Partanen J, Ukkonen E, Taipale J: Genome-wide prediction of mammalian enhancers based on analysis of transcription-factor binding affinity. Cell 2006, I 24:47-59.

12. Krivan W, Wasserman WW: A predictive model for regulatory sequences directing liver-specific transcription. Genome Res 200I, I I:I559-I566.

13. Markstein M, Markstein P, Markstein V, Levine MS: Genome-wide analysis of clustered Dorsal binding sites identifies putative target genes in the Drosophila embryo. Proc Natl Acad Sci USA 2002, 99:763-768.

14. Ochoa-Espinosa A, Yucel G, Kaplan L, Pare A, Pura N, Oberstein A, Papatsenko $D$, Small $S$ : The role of binding site cluster strength in Bicoid-dependent patterning in Drosophila. Proc Natl Acad Sci USA 2005, I 02:4960-4965.

15. Philippakis AA, Busser BW, Gisselbrecht SS, He FS, Estrada B, Michelson AM, Bulyk ML: Expression-guided in silico evaluation of candidate cis regulatory codes for Drosophila muscle founder cells. PLoS Comp Biol 2006, 2:e53.

16. Rebeiz M, Reeves NL, Posakony JW: SCORE: a computational approach to the identification of cis-regulatory modules and target genes in whole-genome sequence data. Site clustering over random expectation. Proc Natl Acad Sci USA 2002, 99:9888-9893.

17. Wasserman WW, Palumbo M, Thompson W, Fickett JW, Lawrence $\mathrm{CE}$ : Human-mouse genome comparisons to locate regulatory sites. Nat Genet 2000, 26:225-228.

18. Wasserman WW, Fickett JW: Identification of regulatory regions which confer muscle-specific gene expression. J Mo Biol 1998, 278: 167-18I.

19. Woolfe A, Goodson M, Goode DK, Snell P, McEwen GK, Vavouri T, Smith SF, North P, Callaway H, Kelly K, et al:: Highly conserved non-coding sequences are associated with vertebrate development. PLOS Biol 2005, 3:e7.

20. Gaudet J, Muttumu S, Horner M, Mango SE: Whole-genome analysis of temporal gene expression during foregut development. PLoS Biol 2004, 2:e352.

21. Wenick AS, Hobert O: Genomic cis-regulatory architecture and trans-acting regulators of a single interneuron-specific gene battery in C elegans. Dev Cell 2004, 6:757-770.

22. Blanchette M, Bataille AR, Chen X, Poitras C, Laganiere J, Lefebvre C, 
Deblois G, Giguere V, Ferretti V, Bergeron D, et al.: Genome-wide computational prediction of transcriptional regulatory modules reveals new insights into human gene expression. Genome Res 2006, 16:656-668.

23. Halfon MS, Michelson AM: Exploring genetic regulatory networks in metazoan development: methods and models. Physiol Genomics 2002, 10:131-143.

24. Bulyk M: Computational prediction of transcription-factor binding site locations. Genome Biol 2003, 5:20I.

25. $\mathrm{Li} \mathrm{H}$, Wang W: Dissecting the transcription networks of a cell using computational genomics. Curr Opin Genet Dev 2003, |3:6||-6|6.

26. Wasserman WW, Sandelin A: Applied bioinformatics for the identification of regulatory elements. Nat Rev Genet 2004, 5:276-287.

27. Michelson AM, Bulyk ML: Biological code breaking in the 2 Ist century. Mol Syst Biol 2006, 2:. 2006.0018

28. Siggia ED: Computational methods for transcriptional regulation. Curr Opin Genet Dev 2005, I5:214-22I.

29. Plessy C, Dickmeis T, Chalmel F, Strahle U: Enhancer sequence conservation between vertebrates is favoured in developmental regulator genes. Trends Genet 2005, $21: 207-210$.

30. Hardison RC: Conserved non-coding sequences are reliable guides to regulatory elements. Trends Genet 2000, 16:369-372.

31. Arnone MI, Davidson EH: The hardwiring of development: organization and function of genomic regulatory systems. Development 1997, 124:185I-1864.

32. Davidson EH, Rast JP, Oliveri P, Ransick A, Calestani C, Yuh C-H, Minokawa T, Amore G, Hinman V, Arenas-Mena C, et al.: A genomic regulatory network for development. Science 2002, 295: 1669-1678.

33. Schroeder MD, Pearce M, Fak J, Fan H, Unnerstall U, Emberly E, Rajewsky N, Siggia ED, Gaul U: Transcriptional control in the segmentation gene network of Drosophila. PLoS Biol 2004, 2:e27I.

34. Lifanov AP, Makeev VJ, Nazina AG, Papatsenko DA: Homotypic regulatory clusters in Drosophila. Genome Res 2003, 13:579-588.

35. Wolff C, Pepling M, Gergen P, Klingler M: Structure and evolution of a pair-rule interaction element: runt regulatory sequences in D. melanogaster and D. virilis. Mech Dev 1999, 80:87-99.

36. Klingler M, Soong J, Butler B, Gergen JP: Disperse versus compact elements for the regulation of runt Stripes in Drosophila. Dev Biol 1996, 177:73-84.

37. Gallo SM, Li L, Hu Z, Halfon MS: REDfly: a Regulatory Element Database for Drosophila. Bioinformatics 2006, 22:38I-383.

38. Moriyama EN, Hartl DL: Codon usage bias and base composition of nuclear genes in Drosophila. Genetics 1993, 134:847-858

39. Bergman C, Pfeiffer B, RincÛn-Limas D, Hoskins R, Gnirke A, Mungall C, Wang A, Kronmiller B, Pacleb J, Park S, et al.: Assessing the impact of comparative genomic sequence data on the functional annotation of the Drosophila genome. Genome Biol 2002, 3:RESEARCH0086.

40. Fitzgerald PC, Sturgill D, Shyakhtenko A, Oliver B, Vinson C: Comparative genomics of Drosophila and human core promoters. Genome Biol 2006, 7:R53.

4l. Bergman CM, Carlson JW, Celniker SE: Drosophila DNase I footprint database: a systematic genome annotation of transcription factor binding sites in the fruitfly, Drosophila melanogaster. Bioinformatics 2005, 2 I:1747- 1749.

42. Sironi M, Menozzi G, Comi GP, Cagliani R, Bresolin N, Pozzoli U: Analysis of intronic conserved elements indicates that functional complexity might represent a major source of negative selection on non-coding sequences. Hum Mol Genet 2005, | 4:2533-2546.

43. Haddrill PR, Charlesworth B, Halligan DL, Andolfatto P: Patterns of intron sequence evolution in Drosophila are dependent upon length and GC content. Genome Biol 2005, 6:R67.

44. Dickmeis T, Muller F: The identification and functional characterisation of conserved regulatory elements in developmental genes. Brief Funct Genomic Proteomic 2005, 3:332-350.

45. Powell JR: Progress and Prospects in Evolutionary Biology: The Drosophila Model USA: Oxford University Press; 1997.

46. Morgenstern B: DIALIGN 2: improvement of the segment-tosegment approach to multiple sequence alignment. Bioinformatics 1999, I 5:21।-218.

47. Pollard DA, Bergman CM, Stoye J, Celniker SE, Eisen MB: Benchmarking tools for the alignment of functional non-coding DNA. BMC Bioinformatics 2004, 5:6.
48. Ludwig MZ, Bergman C, Patel NH, Kreitman M: Evidence for stabilizing selection in a eukaryotic enhancer element. Nature 2000, 403:564-567.

49. Costas J, Casares F, Vieira J: Turnover of binding sites for transcription factors involved in early Drosophila development. Gene 2003, 310:215-220.

50. Dermitzakis ET, Bergman CM, Clark AG: Tracing the evolutionary history of Drosophila regulatory regions with models that identify transcription factor binding sites. Mol Biol Evol 2003, 20:703-7|4

5I. Halligan DL, Keightley PD: Ubiquitous selective constraints in the Drosophila genome revealed by a genome-wide interspecies comparison. Genome Res 2006, 16:875-884.

52. Papatsenko D, Kislyuk A, Levine M, Dubchak I: Conservation patterns in different functional sequence categories of divergent Drosophila species. Genomics 2006, 88:43I-442.

53. Siepel A, Bejerano G, Pedersen JS, Hinrichs AS, Hou M, Rosenbloom K, Clawson H, Spieth J, Hillier LW, Richards S, et al.: Evolutionarily conserved elements in vertebrate, insect, worm, and yeast genomes. Genome Res 2005, I 5:1034-1050.

54. Bejerano G, Pheasant M, Makunin I, Stephen S, Kent WJ, Mattick JS, Haussler D: Ultraconserved elements in the human genome. Science 2004, 304: 1321-1325.

55. Glazov EA, Pheasant M, McGraw EA, Bejerano G, Mattick JS: Ultraconserved elements in insect genomes: $A$ highly conserved intronic sequence implicated in the control of homothorax mRNA splicing. Genome Res 2005, I 5:800-808.

56. The FANTOM Consortium, Carninci $P$, Kasukawa $T$, Katayama $S$, Gough J, Frith MC, Maeda N, Oyama R, Ravasi T, Lenhard B, et al.: The transcriptional landscape of the mammalian genome. Science 2005, 309:1559-1563.

57. Kapranov P, Cawley SE, Drenkow J, Bekiranov S, Strausberg RL, Fodor SPA, Gingeras TR: Large-scale transcriptional activity in chromosomes 2 I and 22. Science 2002, 296:916-919.

58. Stolc V, Gauhar Z, Mason C, Halasz G, van Batenburg MF, Rifkin SA, Hua S, Herreman T, Tongprasit W, Barbano PE, et al.: A gene expression map for the euchromatic genome of Drosophila melanogaster. Science 2004, 306:655-660.

59. Lee S, Bao J, Zhou G, Shapiro J, Xu J, Shi RZ, Lu X, Clark T, Johnson $D$, Kim YC, et al:: Detecting novel low-abundant transcripts in Drosophila. RNA 2005, II:939-946.

60. Yamada K, Lim J, Dale JM, Chen H, Shinn P, Palm CJ, Southwick AM, Wu HC, Kim C, Nguyen M, et al:: Empirical analysis of transcriptional activity in the Arabidopsis genome. Science 2003, 302:842-846.

6I. Rinn JL, Euskirchen G, Bertone P, Martone R, Luscombe NM, Hartman S, Harrison PM, Nelson FK, Miller P, Gerstein M, et al.: The transcriptional activity of human Chromosome 22. Genes Dev 2003, 17:529-540.

62. Cheng J, Kapranov P, Drenkow J, Dike S, Brubaker S, Patel S, Long J, Stern D, Tammana H, Helt G, et al: Transcriptional maps of 10 human chromosomes at 5-nucleotide resolution. Science 2005, 308: | | 49-II54.

63. Johnson JM, Edwards S, Shoemaker D, Schadt EE: Dark matter in the genome: evidence of widespread transcription detected by microarray tiling experiments. Trends Genet 2005, $21: 93-102$.

64. Manak JR, Dike S, Sementchenko V, Kapranov P, Biemar F, Long J, Cheng J, Bell I, Ghosh S, Piccolboni A, et al.: Biological function of unannotated transcription during the early development of Drosophila melanogaster. Nat Genet 2006, 38: I I5I-II58.

65. Abnizova I, te Boekhorst R, Walter K, Gilks WR: Some statistical properties of regulatory DNA sequences, and their use in predicting regulatory regions in the Drosophila genome: the fluffy-tail test. BMC Bioinformatics 2005, 6:109.

66. Chan BY, Kibler D: Using hexamers to predict cis-regulatory motifs in Drosophila. BMC Bioinformatics 2005, 6:262.

67. Grad YH, Roth FP, Halfon MS, Church GM: Prediction of similarly acting cis-regulatory modules by subsequence profiling and comparative genomics in Drosophila melanogaster and $D$. pseudoobscura. Bioinformatics 2004, 20:2738-2750.

68. Rajewsky N, Vergassola M, Gaul U, Siggia ED: Computational detection of genomic cis-regulatory modules applied to body patterning in the early Drosophila embryo. BMC Bioinformatics 2002, 3:30.

69. Sinha S, van Nimwegen E, Siggia ED: A probabilistic method to detect regulatory modules. Bioinformatics 2003, I 9(SuppI I):i292-30I. 
70. Sinha S, Tompa M: A statistical method for finding transcription factor binding sites. Proc Int Conf Intell Syst Mol Biol 2000, 8:344-354.

7I. Emberly E, Rajewsky N, Siggia ED: Conservation of regulatory elements between two species of Drosophila. BMC Bioinformatics 2003, 4:57.

72. Nelson CE, Hersh BM, Carroll SB: The regulatory content of intergenic DNA shapes genome architecture. Genome Biol 2004, 5:R25.

73. Petrov DA, Lozovskaya ER, Hartl DL: High intrinsic rate of DNA loss in Drosophila. Nature 1996, 384:346-349.

74. Babak T, Blencowe BJ, Hughes TR: A systematic search for new mammalian non-coding RNAs indicates little conserved intergenic transcription. BMC Genomics 2005, 6: 104.

75. Bae E, Calhoun VC, Levine M, Lewis EB, Drewell RA: Characterization of the intergenic RNA profile at abdominal-A and abdominal-B in the Drosophila bithorax complex. Proc Natl Acad Sci USA 2002, 99: 16847-16852.

76. Drewell RA, Bae E, Burr J, Lewis EB: Transcription defines the embryonic domains of cis-regulatory activity at the Drosophila bithorax complex. Proc Natl Acad Sci USA 2002, 99:16853-16858.

77. Ashe HL, Monks J, Wijgerde M, Fraser P, Proudfoot NJ: Intergenic transcription and transinduction of the human beta-globin locus. Genes Dev 1997, I I:2494-2509.

78. Ho Y, Elefant F, Liebhaber SA, Cooke NE: Locus control region transcription plays an active role in long-range gene activation. Mol Cell 2006, 23:365-375.

79. Masternak K, Peyraud N, Krawczyk M, Barras E, Reith W: Chromatin remodeling and extragenic transcription at the MHC class II locus control region. Nat Immunol 2003, 4: I32-I37.

80. Rogan DF, Cousins DJ, Santangelo S, loannou PA, Antoniou M, Lee TH, Staynov DZ: Analysis of intergenic transcription in the human IL-4/IL-I 3 gene cluster. Proc Natl Acad Sci USA 2004, I0I:2446-245I.

8I. Johnson KD, Grass JA, Park C, Im H, Choi K, Bresnick EH: Highly restricted localization of RNA polymerase II within a locus control region of a tissue-specific chromatin domain. Mol Cell Biol 2003, 23:6484-6493.

82. Schmitt S, Prestel M, Paro R: Intergenic transcription through a Polycomb group response element counteracts silencing. Genes Dev 2005, 19:697-708.

83. Halfon MS: (Re)modeling the transcriptional enhancer. Nat Genet 2006, 38: I102-I I03.

84. Driever W, Thoma G, Nusslein-Volhard C: Determination of spatial domains of zygotic gene expression in the Drosophila embryo by the affinity of binding sites for the bicoid morphogen. Nature 1989, 340:363-367.

85. Struhl G, Struhl K, Macdonald PM: The gradient morphogen bicoid is a concentration-dependent transcriptional activator. Cell 1989, 57:1259-1273.

86. Rivera-Pomar R, Jackle H: From gradients to stripes in Drosophila embryogenesis: filling in the gaps. Trends Genet 1996, I 2:478-483.

87. Ashe $H$, Mannervik M, Levine M: Dpp signaling thresholds in the dorsal ectoderm of the Drosophila embryo. Development 2000, 127:3305-33/2

88. Lecuit T, Brook WJ, Ng M, Calleja M, Sun H, Cohen SM: Two distinct mechanisms for long-range patterning by Decapentaplegic in the Drosophila wing. 1996, 381:387-393.

89. Ashe HL, Briscoe J: The interpretation of morphogen gradients. Development 2006, 133:385-394.

90. Mann RS, Carroll SB: Molecular mechanisms of selector gene function and evolution. Curr Opin Genet Dev 2002, I 2:592-600.

91. Prabhakar S, Poulin F, Shoukry M, Afzal V, Rubin EM, Couronne O, Pennacchio LA: Close sequence comparisons are sufficient to identify human cis-regulatory elements. Genome Res 2006, 16:855-863.

92. Senger K, Armstrong GW, Rowell WJ, Kwan JM, Markstein M, Levine M: Immunity regulatory DNAs share common organizational features in Drosophila. Mol Cell 2004, 13:19-32.

93. Beer MA, Tavazoie S: Predicting gene expression from sequence. Cell 2004, II 1:185-198.

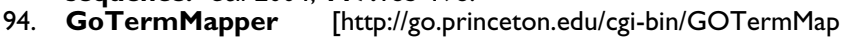
per]

95. Berkeley Comparative Genomics Project [http://hanu man.math.berkeley.edu/genomes/]

96. Genome Sequencing Center, Washington University School of Medicine [http://genome.wustl.edu/]

97. Agencourt Bioscience Corporation [http://www.agen court.com]

98. Richards S, Liu Y, Bettencourt BR, Hradecky P, Letovsky S, Nielsen R, Thornton K, Hubisz MJ, Chen R, Meisel RP, et al:: Comparative genome sequencing of Drosophila pseudoobscura: Chromosomal, gene, and cis-element evolution. Genome Res 2005, 15:1-18.

99. Benson G: Tandem repeats finder: a program to analyze DNA sequences. Nucleic Acids Res 1999, 27:573-580. 